\title{
Aortic response to balloon-injury in the obese Zucker rat: a translational animal model for endovascular interventions
}

\author{
Ludwig D. Orozco ${ }^{1 *}$, Huiling Liu', Betty B. Chen', Razvan F. Buciuc ${ }^{1,2}$, Jonathan D. Fratkin ${ }^{3}$, Juan C. Pisarello', Eddie Perkins ${ }^{1,4}$
}

\begin{abstract}
Background: The small diameter of the carotid balloon-injury (BI) model impedes the evaluation of available endovascular devices. We developed an endovascular BI model in the rat's descending aorta that is compatible with available endovascular instruments. This study also tested the hypothesis that neointimal formation is enhanced in the aorta of obese Zucker (OZ) rats.

Methods: Left external carotid arteriotomies and $\mathrm{BI}$ of the thoracic and abdominal aorta were performed using a Gateway balloon catheter. Aortograms and aortic pathology were examined at 2, 4 and 10 weeks.

Results: Ten weeks after BI the OZ abdominal aorta narrowed $8.34 \pm 1.10 \%$ vs. an expansion of $2.36 \pm 2.24 \%$ in the lean rat (LZ) $(p<0.001)$. Simultaneously, the LZ thoracic aorta expanded $9.50 \pm 4.27 \%$ vs. a stenosis of $2.78 \pm 1.65$ $\%$ in the OZ ( $p=0.003)$. A significant increase in neointimal formation, as measured by the intima to media (l:M) ratio, was observed in the $\mathrm{OZ}$ descending aorta $(\mathrm{p}<0.001)$.

Conclusions: This is a minimally invasive $\mathrm{BI}$ model to the rat's descending aorta compatible with available endovascular instruments. Following $\mathrm{BI}$ the $\mathrm{OZ}$ descending aorta demonstrates enhanced neointimal formation and constrictive vascular remodeling.
\end{abstract}

\section{Background}

A great number of endovascular interventions, either cardiovascular or neurovascular, involve balloon-angioplasty and/or stenting of stenosed or vasospastic arteries. It is well known that balloon injury (BI) to the arterial wall induces restenosis with a typical decrease in lumen diameter as a result of intimal hyperplasia $(\mathrm{IH})$ and vessel wall remodeling $[1,2,3]$. This restenosis occurs more frequently in patients with other cardiovascular risk factors including diabetes, dyslipidemia, obesity and hypertension [4,5]. Zucker rats are frequently utilized as an animal model of obesity, dyslipidemia and type 2-diabetes $[6,7,8]$. The rat common carotid artery $\mathrm{BI}$ model is widely applied to study molecular mechanisms and the role of smooth muscle cells in arterial disease and healing. Apart from this model, data about injury in other arteries are not extensive [1]. From a clinical perspective, the rat carotid model is too small to test available endovascular devices. Other rat models include the infra-renal aorta and the common iliac artery $[1,9,10,11]$. The aortic model requires an open laparotomy, while the common iliac artery is still a small caliber vessel. The goal of the present study was to create a less invasive BI model in the rat's descending aorta. This model is compatible with many endovascular instruments and implantable devices, such as stents, used in clinical practice.

\footnotetext{
*correspondence: lorozco-castillo@umc.edu

${ }^{1}$ Department of Neurosurgery, University of Mississippi Medical Center, 2500 N State Street Jackson, MS, USA.

Full list of author information is available at the end of the article
}

\section{Methods \\ Study design}

Ten-week old, lean (LZ) $(n=21)$ and obese $(O Z)(n=21)$ male Zucker diabetic rats were obtained from Genetic Models (Indianapolis, IN, USA). At 12 weeks of age, endovascular balloon injury of the aorta was induced in the supra-renal abdominal and the thoracic aorta. Rats were randomly subdivided into three post-balloon injury study groups: two weeks $(L Z=6, O Z=6)$, four weeks $(L Z=6, O Z=6)$ and ten weeks $(L Z=9, O Z=9)$.

The animals had free access to rat chow (Harlan laboratories, Indianapolis, IN, USA) and tap water acidified to $\mathrm{pH}$ 4.0. They were maintained at constant humidity $(60+/-5 \%)$, temperature $(24+/-1$ degree $C$ ) and light cycle (06:00 to 18:00 hours). All protocols were approved by the Institutional Animal Care and Use Committee at the University of Mississippi Medical Center and were consistent with the Guide for the care and Use of Laboratory Animals (NIH publication 85-23, revised 1996).

\section{Pre-operative procedure}

Inhalational anesthesia was induced with isoflurane as previously described [12].

\section{Surgical technique}

The left carotid artery was chosen to introduce the balloon-catheter, since it provides a direct access into the descending aorta. Doing this avoids the difficulty of navigating the more rigid balloon catheter system through the aortic arch, as is the case in a right carotid approach (Figure 1). 
A left paramedian neck incision was performed and the skin, sternocleidomastoid and omo/thyrohyoid muscles were retracted with 3-0 vicryl sutures (Ethicon, Inc., Somerville, NJ, USA). Then, under microscopic magnification, the left common, internal and external carotid arteries were exposed. The external carotid artery was ligated with a 5-0 silk suture (Ethicon, Inc., Somerville, NJ USA), 6 mm distal to the carotid bifurcation. The proximal common and internal carotid arteries (including the occipital artery) were temporarily clamped with 5-0 silk suture loops. The superior thyroid artery branching off the proximal external carotid artery was coagulated with bipolar cautery. Lidocaine (Abbott Laboratories, Abbott Park, IL, USA) (5 $\mathrm{mg} / \mathrm{kg}$ ) was applied to prevent vasospasm.

\section{Endovascular technique}

A deflated $3.25 \mathrm{~mm}$ (diameter) by $9 \mathrm{~mm}$ (length) Gateway PTA balloon catheter (Boston Scientific, Natick, MA, USA) was inserted, through a transverse arteriotomy, into the proximal left external carotid artery, and advanced to the common carotid artery. Next, the temporary clamp on the common carotid artery was loosened, and an Agility-10 microwire (Cordis, Miami, FLA, USA) was navigated, inside the balloon catheter, to the descending aorta. Next, the balloon catheter was advanced, over the wire, to the proximal descending aorta. Baseline thoracic and abdominal aortograms were obtained using the balloon catheter (without the microwire), by injecting 0.6-0.8 ml of undiluted Omnipaque-300 (iohexol) (General Electric Healthcare, Princeton, NJ, USA).

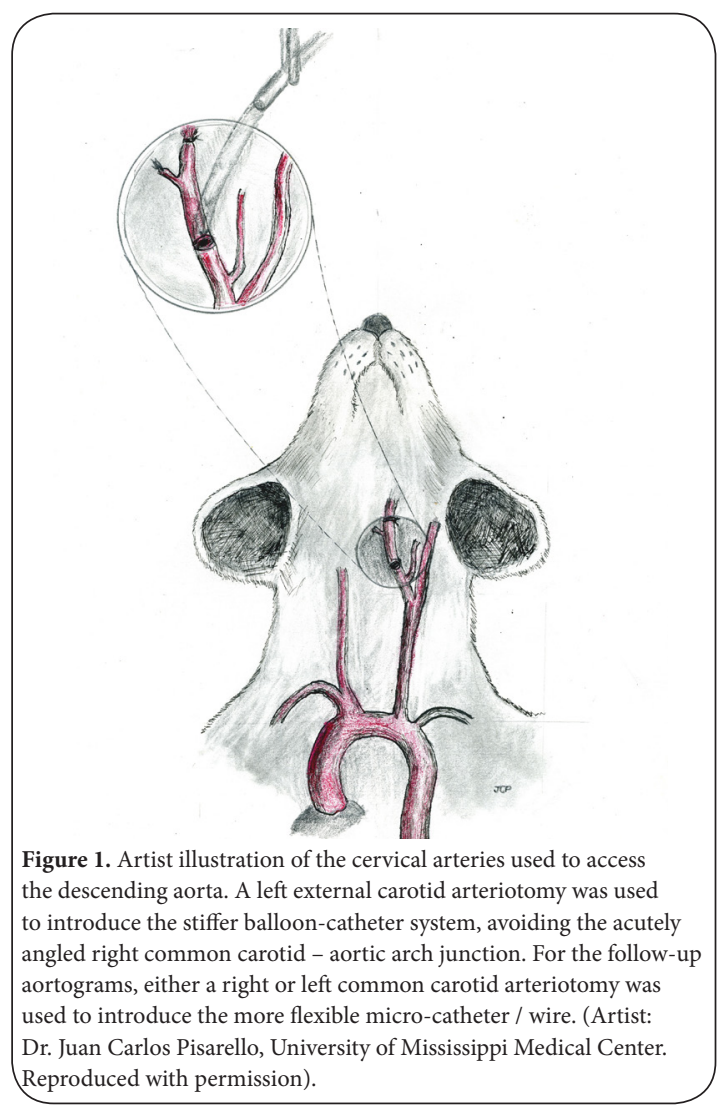
tion.
The abdominal aortic injury was done by advancing the microwire to the infra-renal abdominal aorta, followed by the deflated balloon (over the wire) to just above the renal artery bifurcation. The balloon was inflated at this position with $50 \%$ diluted Omnipaque-300 to a pressure of $2 \mathrm{~atm}$ (manual barometer), and pulled and rotated from distal to proximal, over a $15 \mathrm{~mm}$ segment, for 4 successive times (Figure 2). The thoracic aortic injury was done in similar fashion from just above the diaphragm to a few millimeters distal to the aortic arch, for a $15 \mathrm{~mm}$ segment (Figure 2). This process denudes the vessel of endothelium and disrupts the elastic lamina and underlying smooth muscle cells.

Finally, post-Bl aortograms were obtained to evaluate the injured areas for any immediate complications (dissection, rupture, thrombosis, occlusion) (Figure 2). The balloon catheter was then removed, and the external carotid artery ligated proximal to the arteriotomy with a 5-0 silk suture. The temporary loops were removed from the common and internal carotid arteries to restore perfusion. Neck closure was done in 1 layer of continuous 2-0 vicryl sutures.

\section{Micro-angiography of injured aortic arteries}

At the appropriate follow-up time either left or right paramedian neck incisions were performed, and the common carotid artery was exposed and ligated proximal to the bifurcation with a 5-0 silk suture. A proximal common carotid artery suture loop was applied, and a transverse arteriotomy was performed to allow access of a Prowler Plus microcatheter (Cordis, Miami, FLA, USA)
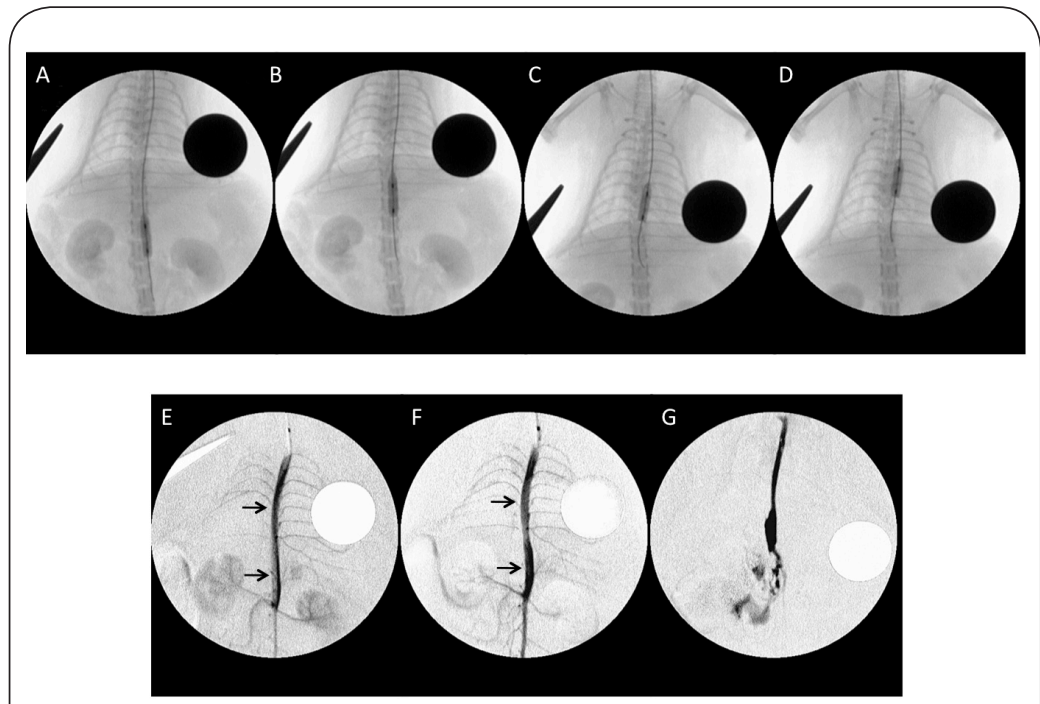

Figure 2. Antero-posterior (A-P) radiographs demonstrate the balloon-injury technique. The balloon was inflated above the renal bifurcation to a pressure of $2 \mathrm{~atm}$ (manual barometer), and then pulled and rotated to just below the diaphragm, four successive times, A and B. The thoracic aortic injury was done in similar fashion from above the diaphragm to a few millimeters distal to the aortic arch, $\mathrm{C}$ and D. The radiopaque circle represents a $19 \mathrm{~mm}$ coin. Pre- (E) and Post- (F) balloon-injury aortograms demonstrate initial dilatation of the injured thoracic and abdominal aorta (arrows). Occasionally, the induced injury would be complicated by aortic dissection ( 5 out of 42 injured animals (11.9\%)) and rupture (extravasation) ( 1 out of 42 injured animals $(2.4 \%)$ ) as seen in G. These complications occur as the result of balloon inflation, vessel overdistension, and increased balloon-vessel wall traction/fric- 
with an Agility-10 microwire. This microcatheter-wire system is more flexible and easier to navigate than the Gateway ballooncatheter. This allows its use through a more angled right carotid approach (Figure 1). Next, the microcatheter was advanced, over the wire, to the proximal descending aorta and thoraco-abdominal aortograms obtained (Figure 3). The microcatheter and wire were removed and the animal euthanized.

Isite picture archiving and communication system (PACS) (Koninklijke Philips, Eindhoven, Netherlands) was used to analyze the pre- and post-injury aortograms. There were two important factors to consider when comparing the angiographic diameters of the aorta. First, is the anatomical taper of the descending aorta with a larger proximal thoracic aorta that tapers down around the diaphragm. It becomes larger again at the renal artery bifurcation (Figure 3). Second, is the increasing aortic size as the animals aged and gained weight [13]. To correct for this variable aortic diameter, we calculated the baseline taper percentage and the post-injury stenosis/expansion percentages. These were obtained by adapting the NASCET [14] formula.

\section{Histomorphometry of injured aortic arteries}

The entire aorta was harvested and the adipose and adventitial layers were surgically removed. These vessels were placed in $2 \%$ paraformaldehyde (Sigma-Aldrich, St. Louis, MO, USA) solution in PBS $(\mathrm{g} / \mathrm{ml})$ for 24 hours, followed by ethanol dehydration and paraffin embedding. Thin cross-sections of 10 micrometers at an interval of $1 \mathrm{~mm}$ were obtained from the injured and uninjured segments, to serve as controls. The sections were mounted on a microscopic slide and stained with hematoxylin and eosin, and trichrome stain (Leica Microsystems, Buffalo Grove, IL, USA).

A computer imaging system equipped with Metamorph V6.37 (Molecular Devices, Sunnyvale, CA, USA) was used to perform histomorphometric analysis of sectioned vessels. Media and intimal thickness were measured at the point of maximal intimal hyperplasia. The media was limited by the internal and external elastic laminae. The intima was measured between the internal elastic lamina and the vessel lumen. The intima to media (I:M) thickness ratio was calculated and used as an index of vascular injury.

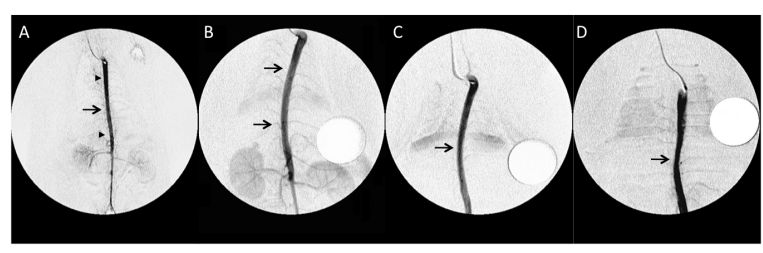

Figure 3. Baseline and post-balloon injury antero-posterior aortograms. At baseline, there is a larger proximal thoracic aorta (upper arrowhead in A) that tapers down around the diaphragm (arrow in A), and becomes larger again at the renal artery bifurcation (lower arrowhead in A), A, non-magnified image. Four and ten weeks after balloon injury the thoracic and abdominal aorta of the LZ rat expanded when compared to baseline (arrows in B, x2 magnified image). Four (C) and ten (D) weeks after injury the abdominal aorta of the $\mathrm{OZ}$ rat developed stenosis (arrows in $\mathrm{C}$ and $\mathrm{D}, \mathrm{x} 2$ magnified images) when compared to baseline.

\section{Statistical methods}

All results are reported as the mean +/- s.e.m. Statistical analysis was performed using SigmaPlot v. 11 software (Systat software Inc. Chicago, IL, USA). Differences between the groups were assessed by two-way analysis of variance (ANOVA) with post hoc testing (StudentNewman-Keuls test). The relationship between angiographic and histologic changes was assessed by Pearson's correlation. A p-value $<0.05$ was considered to be statistically significant.

\section{Results}

At the time of follow-up, all animals appeared healthy. Body weight was significantly greater in $\mathrm{OZ}$ compared to $\mathrm{LZ}$ rats. Five animals died perioperative as a result of abdominal $(L Z n=3, O Z n=1)$ or thoracic $(L Z n=1)$ aortic injury including dissection, thrombosis or rupture (Figure 1). Two animals $(\mathrm{OZ} n=2)$ developed paraplegia as a result of spinal cord ischemia and died three and seven days post-balloon injury, respectively.

\section{Lumen diameter}

The angiographic data is shown in Table 1. The baseline diameter of the thoracic aorta was $2.25 \pm 0.07 \mathrm{~mm}$ and $2.41 \pm 0.09 \mathrm{~mm}$ in the $\mathrm{LZ}$ and $\mathrm{OZ}$, respectively. The baseline diameter of the abdominal aorta was $2.27 \pm 0.06 \mathrm{~mm}$ and $2.22 \pm 0.03 \mathrm{~mm}$ in the $L Z$ and $O Z$, respectively. Four and ten weeks following $\mathrm{BI}$, the thoracic aorta of the $\mathrm{LZ}$ rat expanded $13.30 \pm 9.29 \%$ and $9.50 \pm 4.27 \%$, respectively. This contrasted with an expansion of $1.02 \pm 2.91 \%(p=0.016)$ at 4 weeks, and a stenosis of $2.78 \pm 1.65 \%(p=0.003)$ at 10 weeks seen in the $\mathrm{OZ}$ thoracic aorta. At 2 weeks post-BI, there were no significant differences in the thoracic aortograms of the OZ and LZ rats $(p=0.585)$. Two weeks post-BI the abdominal aorta of the $\mathrm{OZ}$ rat had expanded or remained unchanged $(2.22 \pm 2.77 \%)$ compared to an initial narrowing of $5.59 \pm 1.78 \%(p=0.044)$ in the LZ rat. At 4 weeks the abdominal aorta of the $\mathrm{OZ}$ rat had narrowed $6.88 \pm 3.64 \%$ compared to an expansion of $0.40 \pm 1.33 \%(p=0.036)$ in the $L Z$ rat. Furthermore, 10 weeks after $\mathrm{BI}$ the abdominal aorta of the $\mathrm{OZ}$ rat had narrowed $8.34 \pm 1.10 \%$ compared to an expansion of 2.36 $\pm 2.24 \%$ in the LZ rat $(p<0.001)$ (Figure 3 ).

\section{Intimal hyperplasia}

Visual examination by light microscopy revealed a highly irregular neointima formation in both the abdominal and thoracic aorta of $\mathrm{OZ}$ and LZ rats after balloon-injury (Figure 4). There was increased neointima formation in the abdominal and thoracic aortas of $\mathrm{OZ}$ compared to $\mathrm{LZ}$ at 2, 4, and 10 weeks post-injury (Table 1 and Fig 5). This was associated with a significant increase in the I:M thickness ratio in the $\mathrm{OZ}$ aorta compared to $\mathrm{LZ}$ at 4 weeks $(\mathrm{OZ}$ thoracic $=0.39$ \pm 0.11 vs. $L Z$ thoracic $=0.04 \pm 0.04, p=0.001 .0 Z$ abdominal $=0.54 \pm$ 0.07 vs. $L Z$ abdominal $=0.17 \pm 0.05, p=0.008$ ), and 10 weeks post-BI $(\mathrm{OZ}$ thoracic $=0.39 \pm 0.05 \mathrm{vs} . \mathrm{LZ}$ thoracic $=0.10 \pm 0.05, \mathrm{p}<0.001 . \mathrm{OZ}$ abdominal $=0.70 \pm 0.07$ vs. $L Z$ abdominal $=0.26 \pm 0.11, p<0.001$ ) No correlation was found between the degree of angiographic stenosis, in the injured $\mathrm{OZ}$ thoracic and abdominal aorta, and the 
Table 1. Angiographic and histomorphometric analysis of injured descending aortas from lean (LZ) and obese Zucker (OZ) rats.

Thoracic Aorta

\begin{tabular}{|c|c|c|c|c|c|c|c|}
\hline & $\begin{array}{c}\text { Baseline } \\
\text { Diameter } \\
(\mathrm{mm})\end{array}$ & $\begin{array}{c}\text { Post-injury } \\
\text { Diameter } \\
(\mathrm{mm})\end{array}$ & $\begin{array}{c}\text { Stenosis } \\
\%\end{array}$ & $\begin{array}{c}\text { Expansion } \\
\%\end{array}$ & $\begin{array}{c}\text { Medial } \\
\text { thickness } \\
(\mu \mathrm{m})\end{array}$ & $\begin{array}{c}\text { Peak intimal } \\
\text { thickness } \\
(\mu \mathrm{m})\end{array}$ & I:M thickness \\
\hline \multicolumn{8}{|l|}{ At 2 weeks } \\
\hline $\mathrm{LZ}(\mathrm{n}=4)$ & $2.32 \pm 0.10$ & $2.64 \pm 0.03$ & --- & $1.76 \pm 1.97$ & $85.9 \pm 2.0$ & $7.6 \pm 4.6$ & $0.09 \pm 0.05$ \\
\hline $\mathrm{OZ}(\mathrm{n}=4)$ & $2.61 \pm 0.04$ & $3.00 \pm 0.06$ & --- & $4.73 \pm 1.03$ & $105.4 \pm 2.0$ & $29.0 \pm 6.5$ & $0.27 \pm 0.05$ \\
\hline \multicolumn{8}{|l|}{ At 4 weeks } \\
\hline $\mathrm{LZ}(\mathrm{n}=5)$ & $2.35 \pm 0.03$ & $2.69 \pm 0.09$ & --- & $13.30 \pm 9.29$ & $91.1 \pm 0.2$ & $3.3 \pm 3.3$ & $0.04 \pm 0.04$ \\
\hline $\mathrm{OZ}(\mathrm{n}=5)$ & $2.29 \pm 0.14$ & $2.78 \pm 0.12$ & -- & $1.02 \pm 2.91$ & $113.6 \pm 5.4$ & $45.1 \pm 14.3$ & $0.39 \pm 0.11$ \\
\hline \multicolumn{8}{|l|}{ At 10 weeks } \\
\hline $\mathrm{LZ}(\mathrm{n}=8)$ & $2.07 \pm 0.07$ & $2.77 \pm 0.08$ & --- & $9.47 \pm 4.27$ & $103.2 \pm 2.8$ & $10.4 \pm 5.2$ & $0.10 \pm 0.05$ \\
\hline $\mathrm{OZ}(\mathrm{n}=9)$ & $2.33 \pm 0.08$ & $2.83 \pm 0.06$ & $2.78 \pm 1.65$ & --- & $99.0 \pm 4.0$ & $38.6 \pm 6.1$ & $0.39 \pm 0.05$ \\
\hline \multicolumn{8}{|c|}{ Abdominal Aorta } \\
\hline & $\begin{array}{l}\text { Baseline } \\
\text { Diameter } \\
(\mathbf{m m})\end{array}$ & $\begin{array}{c}\text { Post-injury } \\
\text { Diameter } \\
(\mathbf{m m})\end{array}$ & $\begin{array}{c}\text { Stenosis } \\
\%\end{array}$ & $\begin{array}{c}\text { Expansion } \\
\%\end{array}$ & $\begin{array}{c}\text { Medial } \\
\text { thickness } \\
(\mu \mathrm{m})\end{array}$ & $\begin{array}{c}\text { Peak intimal } \\
\text { thickness } \\
(\mu \mathrm{m})\end{array}$ & I:M thickness \\
\hline \multicolumn{8}{|l|}{ At 2 weeks } \\
\hline $\mathrm{LZ}(\mathrm{n}=4)$ & $2.38 \pm 0.06$ & $2.51 \pm 0.08$ & $5.59 \pm 1.78$ & --- & $98.5 \pm 9.5$ & $12.3 \pm 4.1$ & $0.13 \pm 0.05$ \\
\hline $\mathrm{OZ}(\mathrm{n}=4)$ & $2.32 \pm 0.01$ & $2.63 \pm 0.17$ & --- & $2.22 \pm 2.77$ & $104.8 \pm 10.9$ & $33.2 \pm 4.8$ & $0.31 \pm 0.01$ \\
\hline \multicolumn{8}{|l|}{ At 4 weeks } \\
\hline $\mathrm{LZ}(\mathrm{n}=5)$ & $2.40 \pm 0.07$ & $2.41 \pm 0.07$ & --- & $0.40 \pm 1.33$ & $102.5 \pm 5.9$ & $17.7 \pm 5.4$ & $0.17 \pm 0.05$ \\
\hline $\mathrm{OZ}(\mathrm{n}=5)$ & $2.09 \pm 0.04$ & $2.32 \pm 0.03$ & $6.88 \pm 3.64$ & --- & $109.6 \pm 6.5$ & $61.3 \pm 12.0$ & $0.54 \pm 0.07$ \\
\hline \multicolumn{8}{|l|}{ At 10 weeks } \\
\hline $\mathrm{LZ}(\mathrm{n}=8)$ & $2.03 \pm 0.04$ & $2.53 \pm 0.07$ & --- & $2.36 \pm 2.24$ & $103.2 \pm 2.8$ & $27.0 \pm 11.6$ & $0.26 \pm 0.11$ \\
\hline $\mathrm{OZ}(\mathrm{n}=9)$ & $2.25 \pm 0.05$ & $2.56 \pm 0.08$ & $8.34 \pm 1.10$ & --- & $117.5 \pm 8.4$ & $83.0 \pm 11.5$ & $0.70 \pm 0.07$ \\
\hline
\end{tabular}

Data are mean +/- s.e.m. Sample size is enclosed in parentheses. Differences between LZ and OZ groups were determined by group wise ANOVA.

amount of neointimal formation, as measured by the I:M thickness ratio, or the peak intimal or medial thickness.

\section{Discussion}

Experimental models in various animal species have been used to study the pathological intimal formation as a response of vessel wall damage. The rat carotid artery balloon-injury is one of the most convenient, rapid and thoroughly investigated models for the assessment and treatment of intimal hyperplasia [10]. However, the carotid artery is too small to test the presently available endovascular devices used in the clinical setting. Furthermore, in contrast to human arteries, the rat carotid artery has no vasa vasorum, contains a much thinner subintimal layer, and a lower elastin / higher collagen content in the tunica media [15].
In this study the larger abdominal and to a lesser extent thoracic aorta were validated as an alternative model to be used for the evaluation of arterial response after endovascular techniques. Currently available endovascular catheters, wires, balloons and stents, provide enhanced access and flexibility. This allows their use in this small animal model. In humans, the composite coronary artery diameter ranges from 1.9 to $5.4 \mathrm{~mm}$ (average $3.4 \pm 0.5 \mathrm{~mm}$ ), and the average diameter of the middle cerebral artery is 3 to $5 \mathrm{~mm}[16,17]$. This corresponds to the baseline diameter of the LZ and $\mathrm{OZ}$ descending aortas. The rat thoracic and abdominal aorta is large enough to deploy, and potentially test, the majority of available coronary and intracranial stents with their delivery systems. 

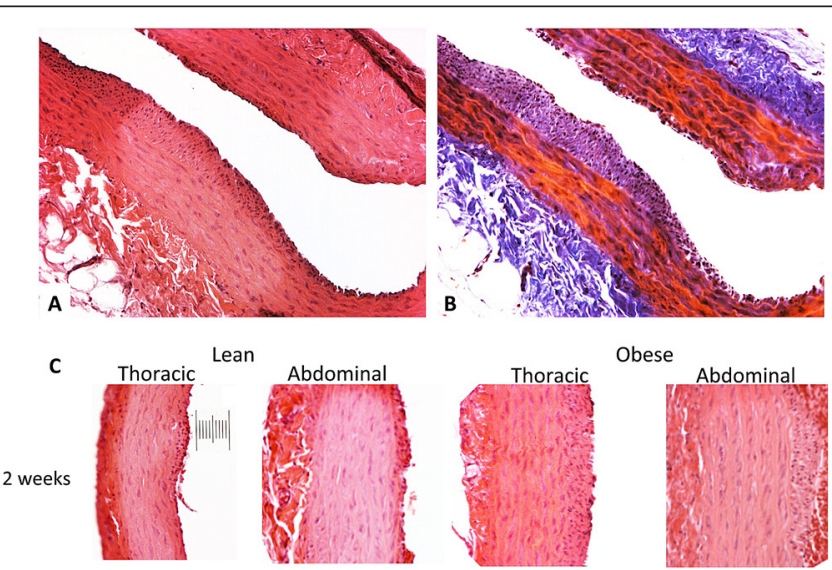

Obese
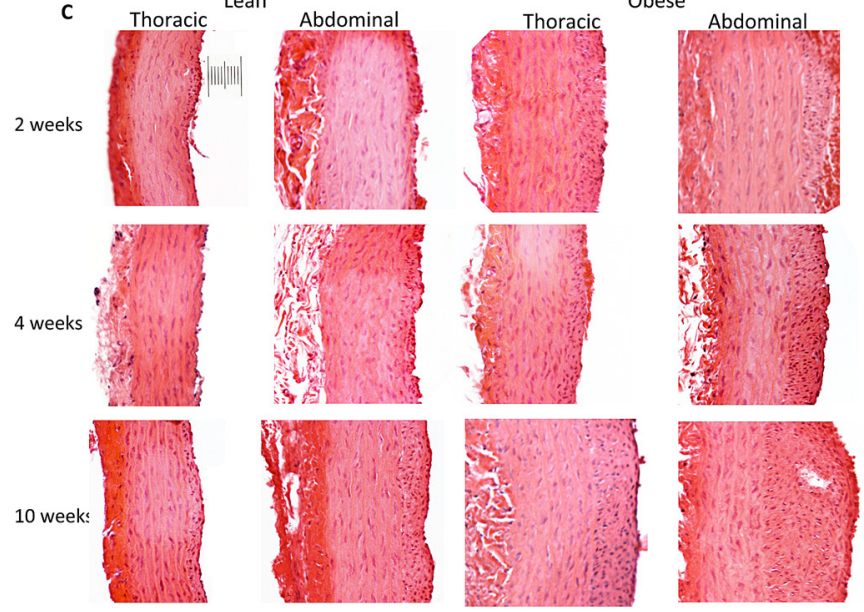

Figure 4. Photographs show HE (A) and trichrome (B) stained cross-section of the descending aorta after balloon injury (BI), demonstrating highly irregular neointima formation, original magnification $\mathrm{x} 100$. This was seen in both the abdominal and thoracic aorta of OZ and LZ rats. Photographs in C show HE-stained cross-section of the thoracic and abdominal aorta in the $\mathrm{LZ}$ and $\mathrm{OZ}$ rat at 2, 4 and 10 weeks after balloon injury (BI), original magnification $\mathrm{x} 40$. These show a temporal increase in neointima formation in the abdominal and thoracic aortas of OZ compared to LZ rats.

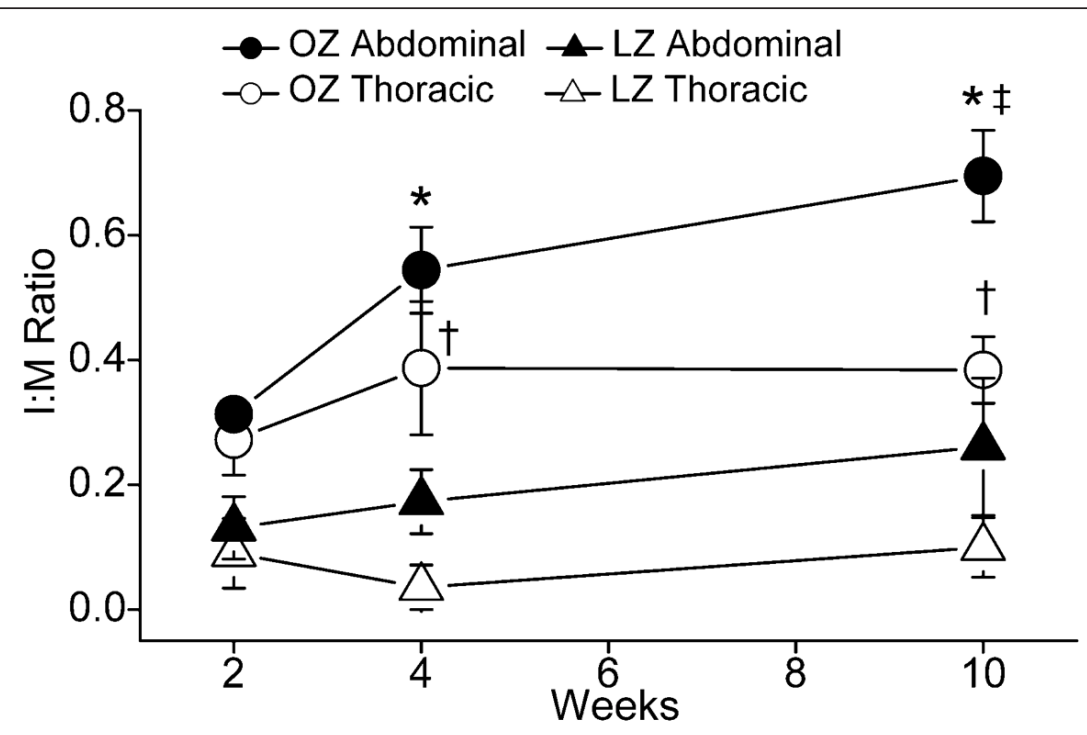

Figure 5. Diagram showing the mean I:M thickness ratio of the OZ and LZ thoracic and abdominal aorta, plotted against time in weeks. The error bars are standard errors of the mean (At 2 weeks $n=4$, at 4 weeks $n=5$ for each $\mathrm{LZ}$ and OZ, and at 10 weeks $\mathrm{n}=8$ for LZ and $\mathrm{n}=9$ for OZ). Type-Time Difference: $\left.{ }^{*}\right)$ OZ vs. LZ abdominal aorta at 2 weeks NS, at 4 weeks $\mathrm{p}=0.008$, at 10 weeks $\mathrm{p}<0.001$. ( $\dagger) \mathrm{OZ}$ vs. LZ thoracic aorta at 2 weeks NS, at 4 weeks $\mathrm{p}=0.001$, at 10 weeks $p<0.001$. Ten weeks ( $\$$ ) after BI the amount of neointimal formation of the OZ abdominal aorta significantly increased when compared to 2 weeks post-BI, $\mathrm{p}=0.012$. 
In our study, the lean thoracic aorta exhibited the least amount of intimal hyperplasia, where 9 of 17 displayed no $\mathrm{IH}$ at different time points. At the same time the lean thoracic aorta developed the largest amount of adaptive remodeling, 4 and 10 weeks after $\mathrm{BI}$, as measured by the percentage of expansion. The lean abdominal aorta exhibited more IH than the lean thoracic aorta, but significantly less than the obese abdominal aorta. The lean abdominal aorta had a trend towards adaptive remodeling 4 and 10 weeks after $\mathrm{BI}$. This is in agreement with prior reports demonstrating that the abdominal aorta of Wistar rats compensate to IH by adaptive remodeling [1]. The obese abdominal aorta responded differently, and developed the largest amount of $\mathrm{IH}$ and constrictive remodeling. Both of these responses increased with time and were significantly different than their lean counterpart. The obese thoracic aorta expanded initially but had a trend to constrict at 10 weeks after balloon-injury. Additionally, the obese thoracic aorta exhibited a significantly larger $\mathrm{IH}$ when compared to their lean counterpart. Previous reports have documented a similar propensity in the obese Zucker rat carotid artery to develop IH following balloon-injury $[13,18]$ Our results not only demonstrate increased neointimal formation in the aorta of the obese animal, but also a propensity towards constrictive remodeling. We did not find a correlation between the degree of angiographic stenosis/expansion and the amount of neointimal formation at any time point. This suggest, as previously reported [19], that vessel wall remodeling and neointima formation are two separate processes. Multiple factors may account for the different $\mathrm{IH}$ and arterial remodeling response observed in the thoracic and abdominal aortas of lean and obese rats after balloon injury. These include: an arterytype dependent variation of both synthetic and contractile smooth muscle cells, the number of vasa vasorum in the tunica adventitia, and the increasingly important role of the tunica adventitia in inflammation, growth and repair $[17,19,20]$.

The abdominal aorta of both lean and obese rats developed $\mathrm{IH}$ that continued to increase up to 10 weeks after injury and perhaps beyond. The thoracic aorta of both animal groups had a lesser degree of $\mathrm{IH}$ that tended to increase with time. This has been demonstrated before and is different than the rat carotid injury model where IH peaks between 14-28 days post-BI [1,21-23]. We observed an irregular or patchy $\mathrm{IH}$ in the descending aorta of the Zucker rat, which is different than the uniformly circumferential response observed in the carotid balloon injury model and the abdominal aorta/common iliac artery models reported elsewhere [1]. Koletsky and Snajdar had previously reported a similar, less uniform and less rapid, response in a rat model of aortic balloon-injury [23]. We hypothesize that this is due to a smaller lumen / balloon ratio (0.6-0.8), the use of the Gateway semi-compliant balloon, as well as the plane of attachment of the descending aorta and its segmental branches, with a free anterior and fixed posterior sides.

\section{Conclusions}

1. This model provides a less invasive access to the rat's descending aorta, and is compatible with many endovascular instruments used in clinical practice. The rat aorta is large enough to potentially deploy and test coronary and intra-cranial stents.

2. Neointimal formation following $\mathrm{BI}$ is enhanced in the abdominal and thoracic aorta of the obese Zucker (OZ) rat.

3. $\mathrm{BI}$ tends to induce constrictive remodeling in the $\mathrm{OZ}$ aorta as opposed to the adaptive remodeling seen in the $L Z$ aorta.

\section{List of abbreviations}

Atm: Atmospheres

BI: Balloon injury

$\mathrm{IH}$ : Intimal hyperplasia

I M: Intima to media

LZ: Lean Zucker rat

NASCET: North American Symptomatic Carotid Endarterectomy Trial OZ: Obese Zucker rat

PACS: Picture archiving and communication system

\section{Competing interests}

The authors report no financial or non-financial competing interests concerning the materials, methods, findings or conclusions specified in this paper.

\section{Authors' contributions}

LO conceived the study, carried out the $\mathrm{Bl}$, angiographic and harvesting experiments, and drafted the manuscript. HL carried out the $\mathrm{Bl}$, angiographic and harvesting experiments, performed the histomorphometric and statistical analyses and processed the images. BC carried out the histopathology and histomorphometric analysis. RB participated in the design of the study and angiographic analysis. JF participated in the histopathology and histomorphometric analysis. JP participated in the $\mathrm{Bl}$ and angiographic experiments and illustration. EP participated in the conception and design, carried out the $\mathrm{Bl}$, angiographic and harvesting experiments, and coordinated the study. All authors read and approved the final manuscript.

\section{Acknowledgements and Funding}

This work was supported by the Departments of Anatomy, Neurosurgery and Radiology at the University of Mississippi Medical Center, Jackson, MS.

\section{Author information}

${ }^{2}$ Department of Radiology, University of Mississippi Medical Center, 2500 N State Street Jackson, MS, USA.

${ }^{3}$ Department of Pathology, University of Mississippi Medical Center, 2500 N State Street Jackson, MS, USA.

${ }^{4}$ Department of Anatomy, University of Mississippi Medical Center, 2500 N State Street Jackson, MS, USA.

\section{Article history}

Editor: A. Mantalaris, Imperial College London, United Kingdom. Received: 7-Jan-2012 Revised: 2-Mar-2012

Accepted: 8-Mar-2012 Published: 19-Mar-2012 


\section{References}

1. Gabeler EE, van Hillegersberg $R$, Statius van Eps RG, Sluiter W, Gussenhoven EJ, Mulder P, et al. : A comparison of balloon injury models of endovascular lesions in rat arteries. BMC CardiovasC Disord 2002; 2;16

2. Glagov S: Intimal hyperplasia, vascular modeling, and the restenosis problem. Circulation 1994; 89; (6.);2888-91

3. Pasterkamp G, de Kleijn DP, Borst C: Arterial remodeling in atherosclerosis, restenosis and after alteration of blood flow: potential mechanisms and clinical implications. Cardiovasc Res 2000; 45; (4.);843-52

4. Hammoud T, Tanguay JF, Bourassa MG: Management of coronary artery disease: therapeutic options in patients with diabetes. J Am Coll Cardiol 2000; 36; (2.);355-65

5. Stamler J, Vaccaro O, Neaton JD, Wentworth $D$ : Diabetes, other risk factors, and 12-yr cardiovascular mortality for men screened in the Multiple Risk Factor Intervention Trial. Diabetes Care 1993; 16;(2.);434-44

6. Mathe $D$ : Dyslipidemia and diabetes: animal models. Diabete Metab 1995; 21;(2.);106-11

7. Paulson DJ, Tahiliani AG: Cardiovascular abnormalities associated with human and rodent obesity. Life Sci 1992; 51;(20.);1557-69

8. Standley PR, Rose KA, Sowers JR: Increased basal arterial smooth muscle glucose transport in the Zucker rat. Am J Hypertens $1995 ; 8 ;(1.) ; 48-52$

9. Winocour PD, Richardson M, Kinlough-Rathbone $\mathrm{RL}$ : Continued platelet interaction with deendothelialized aortae associated with slower re-endothelialization and more extensive intimal hyperplasia in spontaneously diabetic BB Wistar rats. Int J Exp Pathol 1993; 74;(6.);603-13

10. Johnson GJ, Griggs TR, Badimon L: The utility of animal models in the preclinical study of interventions to prevent human coronary artery restenosis: analysis and recommendations. On behalf of the Subcommittee on Animal, Cellular and Molecular Models of Thrombosis and Haemostasis of the Scientific and Standardization Committee of the International Society on Thrombosis and Haemostasis. Thromb Haemost 1999; 81;(5.);835-43

11. Langeveld $B$, Roks AJ, Tio RA, van Boven AJ, van der Want JJ, Henning RH, et al.: Rat abdominal aorta stenting: a new and reliable small animal model for in-stent restenosis. J Vasc Res 2004; 41;(5.);377-86

12. Kissin I, Morgan PL, Smith LR: Comparison of isoflurane and halothane safety margins in rats. Anesthesiology 1983; 58;(6.);556-61

13. Shelton J, Wang D, Gupta H, Wyss JM, Oparil S, et al. (2003) The neointimal response to endovascular injury is increased in obese Zucker rats. Diabetes Obes Metab 5: 415-423
14. Ferguson GG, Eliasziw M, Barr HW, Clagett GP, Barnes RW, Wallace MC, et al.: The North American Symptomatic Carotid Endarterectomy Trial : surgical results in $\mathbf{1 4 1 5}$ patients. Stroke 1999; 30;(9.);1751-8

15. Sims FH: A comparison of structural features of the walls of coronary arteries from 10 different species. Pathology 1989; 21;(2.);115-24

16. Aronson D, Bloomgarden Z, Rayfield EJ: Potential mechanisms promoting restenosis in diabetic patients. J Am Coll Cardiol 1996; 27;(3.);528-35

17. Jain KK: Some Observations on the Anatomy of the Middle Cerebral Artery. Can J Surg 1964; 7;134-9

18. Park SH, Marso SP, Zhou Z, Foroudi F, Topol EJ, et al. Neointimal hyperplasia after arterial injury is increased in a rat model of non-insulin-dependent diabetes mellitus. Circulation 104: 815-819

19. Kundi R, Hollenbeck ST, Yamanouchi D, Herman BC, Edlin $\mathrm{R}$, Ryer EJ, et al.: Arterial gene transfer of the TGFbeta signalling protein Smad3 induces adaptive remodelling following angioplasty: a role for CTGF. Cardiovasc Res 2009; 84;(2.);326-35

20. Goldberg ID, Stemerman MB, Ransil BJ, Fuhro RL In vivo aortic muscle cell growth kinetics. Differences between thoracic and abdominal segments after intimal injury in the rabbit. Circ Res 47: 182-189

21. Clowes AW, Reidy MA, Clowes MM: Mechanisms of stenosis after arterial injury. Lab Invest 1983; 49; (2.);208-15

22. Forte A, Esposito S, De Feo M, Galderisi U, Quarto $C$, Esposito $F$, et al.: Stenosis progression after surgical injury in Milan hypertensive rat carotid arteries. Cardiovasc Res 2003; 60;(3.);654-63

23. Koletsky S, Snajdar RM: Atherosclerosis following balloon catheter injury to the carotid artery and the aorta of hypertensive rats with normolipidemia or hyperlipidemia. Am J Pathol 1981; 103;(1.);10515 